\title{
Yield Loss Assessment of Barley Yellow Dwarf Disease on Spring Oat in Illinois
}

\author{
Ellen M. Bauske, Horticulture Department, Auburn University, Auburn, AL 36849; Suzanne M. Bissonnette, Uni- \\ versity of Illinois Cooperative Extension Service, Urbana 61874; and Adrianna D. Hewings, USDA ARS, 1815 \\ University St., Peoria, IL 61694
}

\begin{abstract}
Bauske, E. M., Bissonnette, S. M., and Hewings, A. D. 1997. Yield loss assessment of barley yellow dwarf disease on spring oat in Illinois. Plant Dis. 81:485-488.

The quantitative relationship between yield of spring oat cvs. Ogle and Noble and incidence of barley yellow dwarf (BYD) disease caused by barley yellow dwarf virus (BYDV-PAV-IL) was studied. Field plots were infested with Rhopalosiphum padi vectoring BYDV-PAV-IL. A linear critical point regression model was developed that related percentage of maximum yield to BYD incidence, assessed by estimating the percentage of plants with BYD symptoms at Feekes growth stage 10.5 in each plot. The model $y=96.9-0.45 x\left(r^{2}=0.53\right)$ best explained the relationship. Test weight was unaffected by BYD. Although Noble consistently sustained higher disease incidence than Ogle, covariate analysis indicated that the effect of BYD on yield was similar for both cultivars. Studies of random versus focus inoculation of Noble oat, designed to determine the importance of compensation in reducing yield loss associated with BYD, indicated that compensation did not play an important role.
\end{abstract}

Barley yellow dwarf (BYD) disease is the most widespread viral disease of small grains (3). BYD is caused by barley yellow dwarf virus (BYDV). BYDV is a group of several closely related luteovirus strains that are transmitted in a circulative, persistent manner by at least 25 aphid species (16). Strains of BYDV are differentiated by the species of aphids that transmit them $(9,18)$. Two strains, PAV vectored by Sitobion avenae (Fabricius) and Rhopalosiphum padi (L.), and RPV vectored by $R$. padi, occur in Illinois (4). PAV is the predominant strain.

Although BYD is economically important throughout the world, there are few quantitative estimates of its impact on yield. One estimate of annual yield losses averaged $3.8 \%$ for oat and $1.6 \%$ for barley in the United States from 1951 to 1960 (3). However, general yield loss estimates cannot be used effectively to assess loss in individual fields. To permit effective estimation of quantitative relationships between increasing levels of disease and yield loss, epidemics representing several levels of disease incidence and severity must be produced (13). Quantitative models have been developed to estimate yield losses caused by BYD disease in wheat under field conditions in Australia (19) and Canada (10). We are unaware of similar work to estimate the effect of BYD on oat yield, although its effects on yield components have been studied (8).

Corresponding author: E. M. Bauske E-mail: ebauske@acesag.auburn.edu

Accepted for publication 30 January 1997.

Publication no. D-1997-0304-04R

(C) 1997 The American Phytopathological Society
Compensation by healthy plants surrounding diseased plants may reduce the effects of viral diseases on yield loss. Reestman (17) reviewed the importance of compensation by healthy plants surrounding diseased plants. Compensation occurs when healthy plants take advantage of space and resources unused by diseased or dead plants. Compensation is more likely to occur if the infected plant is stunted or dies while the rest of the crop is actively growing. Theoretically, there may be little or no effect of disease on yield when compensation is an important factor. Because stunting and reduced tillering are associated with BYDV infection and greater symptom expression is associated with early infection $(6,11)$, compensation may reduce the impact of BYD on spring oat yield.

If compensation occurs, the method of plant inoculation in field plots will affect yield loss. Inoculation creating foci of infection (12) reduces the effects of compensation. A given number of viruliferous aphids placed on two or three adjacent plants can spread the virus by moving from the focus of infection and feeding on plants surrounding the focus. Only a limited number of uninfected plants on the edge of this expanding focus are able to compensate for the infected plants. Conversely, when plants are infected by distributing the same number of aphids evenly over the entire plot, creating a random pattern of infected plants, diseased plants are interspersed with and surrounded by healthy plants. Under these conditions, compensation will reduce yield losses if it is an important factor. If compensation is not a significant factor, no differences in yield loss will be detected between plots inoculated using these methods.

The purpose of this study was to develop a model for estimating yield losses due to BYD disease on spring oat and to determine whether compensation reduces yield losses associated with the disease.

\section{MATERIAL AND METHODS}

BYD yield loss model. Experimental oat plots to estimate yield losses were planted at the University of Illinois Agronomy/ Plant Pathology South Farm at Urbana in 1991 and 1992. Two spring oat (Avena sativa L.) cultivars were used: Noble, which is very susceptible to BYDV, and Ogle, which is highly resistant to BYDV. Each field plot was $1.1 \times 4.3 \mathrm{~m}$ and was planted with $40 \mathrm{~g}$ of seed with a six-row cone-type planter. Experimental plots were separated by border plots of either cv. Hazel or Ogle, both of which are resistant to BYD disease. Standard agronomic practices were followed throughout the studies (20).

Viruliferous $R$. padi were reared on BYDVPAV-IL-infected barley (cv. Hudson) as previously described (12). Plots were inoculated when plants were at Feekes growth stage 2 (14). Aphids mixed with cornmeal at a ratio of $1: 2(\mathrm{vol} / \mathrm{vol})$ were placed in a mechanical infestation device calibrated to release a uniform amount of the cornmealaphid mixture (21). Each discharge resulted in the release of approximately 70 viruliferous aphids on three to four adjacent plants, creating a focus of infection.

Incidence of BYD disease was evaluated by visually estimating the percentage of plants in each plot with BYD symptoms. Symptoms included tissue discoloration, stunting, reduced tillering, and early senescence. Estimates were recorded weekly, beginning when symptom expression became apparent and ending when the majority of plants were at Feekes growth stage 11, after which BYD symptoms were difficult to distinguish from normal senescence.

Infection with BYDV-PAV-IL was verified in field plots by a triple-antibody sandwich enzyme-linked immunosorbent assay (TAS-ELISA) (5). Three symptomatic flag2 leaves were collected from inoculated plots during both years. Samples were collected 3 weeks after inoculation and stored at $-80^{\circ} \mathrm{C}$. A 0.1 -g tissue sample from each leaf was extracted with a leaf-sap extractor, and TAS-ELISA was done. Plates were read automatically at $405 \mathrm{~nm}$ with an ELISA plate reader (Dynatech MR 700, Dynatech Laboratories, McLean, VA). At least three samples of healthy oat tissue were run on each ELISA plate as controls. A sample was considered positive for BYDV-PAV-IL 
if absorbance was greater than two times the mean of the negative control.

In 1991, the trial consisted of six replications of eight treatments arranged in a $2 \times 4$ factorial. Cvs. Ogle and Noble were planted and inoculated with $0,1,2$, or 3 foci, at approximately 70 aphids per focus as described above. In 1992, the experiment had four replications of six treatments BYD disease incidence and reduce the number of plots with trace levels of infection, both cultivars were inoculated with 1,2 , or 3 foci, and noninoculated plots were eliminated from this trial. One week after inoculation, all plots in all trials were sprayed with the systemic insecticide dimethoate (Cygon $4 \mathrm{EC}$ ) at $277 \mathrm{~g}$ a.i./ha to kill the introduced aphids. Plots were evaluated for BYD disease weekly, and the plant growth stage was recorded at each disease assessarranged in a $2 \times 3$ factorial. To increase

ment. Prior to machine harvest, plots were trimmed to a length of $2.4 \mathrm{~m}$. Moisture content was determined, and plot yields and test weights were standardized to a moisture content of $12.5 \%$. Percent maximum yield was calculated for each plot. The intercept $\left(b_{0}\right)$ of the regression equation of yield on disease incidence was considered the best estimate of maximum yield for individual cultivars within trials. Data were analyzed using PC-SAS, version 6.04 HD (SAS Institute, Cary, NC).

Analysis of covariance as outlined by Littell et al. (15) was used to determine whether the yield and test weight of cvs. Ogle and Noble were affected similarly by BYD disease incidence in each trial. Cultivars and replications were treated as class variables, and disease incidence was treated as the covariate (continuous variable). Using this model, the regression of percent

Table 1. Mean and range of barley yellow dwarf (BYD) disease incidence and yield and test weight of two oat cultivars over 2 years in Illinois

\begin{tabular}{|c|c|c|c|c|c|c|}
\hline \multirow{2}{*}{$\begin{array}{l}\text { Cultivar } \\
\text { and year }\end{array}$} & \multicolumn{2}{|c|}{ Disease incidence $(\%)^{\mathrm{a}}$} & \multicolumn{2}{|c|}{ Yield (kg/ha) } & \multicolumn{2}{|c|}{ Test weight $\left(\mathrm{kg} / \mathrm{m}^{3}\right)$} \\
\hline & Mean & Range & Mean & Range & Mean & Range \\
\hline \multicolumn{7}{|l|}{ Noble } \\
\hline 1991 & 15.0 & $0-45$ & 3,724 & $2,655-4,998$ & 474 & $442-509$ \\
\hline 1992 & 69.8 & $15-95$ & 3,179 & $2,127-4,825$ & 445 & $414-476$ \\
\hline \multicolumn{7}{|l|}{ Ogle } \\
\hline 1991 & 9.7 & $0-30$ & 3,703 & $2,833-4,781$ & 476 & $436-515$ \\
\hline 1992 & 12.9 & $2-25$ & 6,299 & $5,488-6,944$ & 446 & $417-474$ \\
\hline
\end{tabular}

a Disease incidence was determined by visually estimating the percentage of plants in each plot with BYD symptoms at Feekes growth stage 10.5.

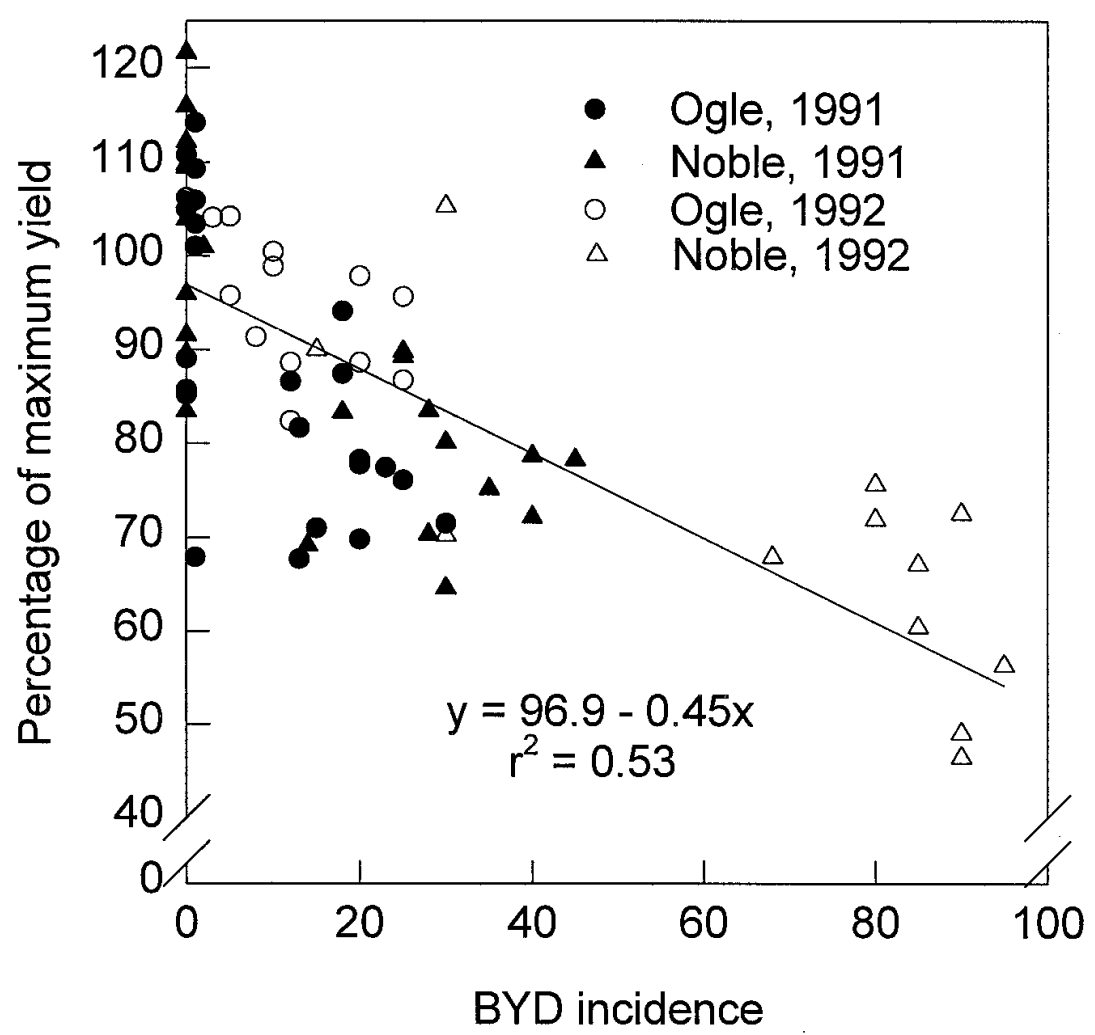

Fig. 1. Effect of barley yellow dwarf (BYD) disease incidence, assessed at Feekes growth stage 10.5, on yield of spring oat cvs. Ogle and Noble. yield or test weight on disease incidence was determined within each cultivar. The test of the covariate by cultivar interaction was a test of homogeneity of slopes between cultivars and determined whether the response of percent yield to BYD incidence was the same for both cultivars.

Analysis of covariance was used to determine whether a generalized model describing yield loss due to BYD disease could be produced using data from both trials. Data were pooled by plant growth stage at disease assessment. Block, cultivars, and years were treated as discrete variables, and BYD incidence was treated as the covariate. Generalized models were created only when significant $(P \leq 0.05)$ main effects of disease were detected and no significant year by disease incidence interactions were detected, indicating that the effect of disease on yield was similar in both years. The model was developed with disease incidence as the independent variable $\left(x_{i}\right)$ by ordinary least squares regression. The significance of the model was evaluated by the $F$ statistic, by the coefficient of determination $\left(r^{2}\right)$, and by determining whether the slope estimate was significantly different from 0 using Student's $t$ statistic.

Residual plots were evaluated to determine whether inconsistencies were present in the generalized model. Inconsistent variance or systematic lack of fit could indicate that transformations of $\hat{Y}$ or $x_{i}$ might be appropriate (7). In addition, the model was evaluated and validated using the prediction sum of squares (PRESS) statistic (1), following procedures outlined by Bissonnette et al. (2). To calculate the PRESS, an observation is left out, and a given model is fit to the remaining data. The eliminated observation is predicted, and the square of the discrepancy between the observed and predicted is calculated. This is repeated for all data points. The PRESS statistic is the sum of these values. Because the expected value of the PRESS statistic is the error variance, it can be used to validate the model. This validation technique is particularly useful in determining whether the deletion of a few critical observations greatly affects the fit of the model (7).

Compensation tests. Cv. Noble was planted in two experiments, one located at Urbana, IL, in 1991 and the other at the University of Illinois DeKalb Experiment Station, DeKalb, in 1992. Field plots were planted and maintained as described above. Experiments were arranged in a randomized complete-block design with five replications of six treatments. The treatments consisted of two inoculation methods (focus or random) and three levels of inoculation. Half of the plots were "focus" inoculated by discharging the infestation device 1,2 , or 3 times in the plot as outlined above. Each discharge released approximately 70 aphids. Foci were separated by at least $0.5 \mathrm{~m}$ in each plot. The other plots 
received a "random" inoculation treatment. The device was discharged 1,2 , or 3 times into a glass jar. The lid of the jar contained 150.3 -cm-diameter holes. The aphids were shaken gently over the entire plot, providing a random distribution of viruliferous aphids throughout the plot. This method resulted in a random distribution of disease.

Disease assessment was made at Feekes growth stages $10,10.5$, and 11 . Plots were harvested as described above. Percent maximum yield was calculated using the intercept $\left(b_{0}\right)$ of the regression equation of yield on disease assessment as the best estimate of maximum yield. Covariance analysis was used to determine the effect of compensation on percent yield. Replication and inoculation methods were treated as class variables, and disease was the covariate. The $F$ test of the interaction of the inoculation method and disease incidence is a test of homogeneity of slopes within inoculation technique. It determines whether the response of yield to BYD incidence is the same for both inoculation techniques. Yield loss models were developed as described above.

\section{RESULTS}

Yield loss model. BYD symptoms were visible in Noble and Ogle plots 2 weeks after inoculation. More than $90 \%$ of the inoculated plots in both trials tested positive for BYDV-PAV-IL with TAS-ELISA.

Disease estimates were taken at growth stages 9, 10.5, and 11. Although plots were treated with Cygon to kill introduced aphids, no effort was made to control wild aphids, and disease incidence increased throughout the growing season. Disease incidence at growth stage 9 did not exceed $7 \%$ in either cultivar in either trial. Therefore, no yield loss models were created or tested for this variable. Symptoms were difficult to distinguish from normal senescence by growth stage 11 , and models created at that growth stage had poor fit and were not considered further.

Generally, symptom expression in 1992 was greater than in 1991 (Table 1). In 1991, Noble and Ogle averaged 15 and $9.7 \%$ disease incidence, respectively, at growth stage 10.5. In 1992, they averaged 69.8 and $12.9 \%$, respectively. Yields also varied among the cultivars in the two trials. In 1991, Noble and Ogle had similar yields: 3,724 and $3,703 \mathrm{~kg} / \mathrm{ha}$, respectively. In 1992, Ogle's yield averaged 6,299 $\mathrm{kg} / \mathrm{ha}$, whereas Noble yielded an average of $3,179 \mathrm{~kg} / \mathrm{ha}$.

Test weight ranged from a low of 414 $\mathrm{kg} / \mathrm{m}^{2}$ for Noble in 1992 to a high of 515 $\mathrm{kg} / \mathrm{m}^{2}$ for Ogle in 1992 (Table 1). There appeared to be no effect of BYD disease on test weight ( $F$ tests of disease main effects were not statistically significant for assessments at any growth stage), and no models were developed based on test weight.

Analysis of each trial indicated that disease incidence estimated when plants were at growth stage 10.5 was significantly re- lated to yield and percent maximum yield as evaluated by the $F$ statistic $(P \leq 0.05)$. In covariance analysis, cultivar by disease interactions were not significant in either trial. Because the response of Noble and Ogle to BYD incidence was similar, a single model was developed for both cultivars.

The results of the analysis of covariance indicated that a generalized model describing percent yield loss due to BYD disease at growth stage 10.5 would be valid. When data from both years were combined, the main effects of disease were significant $(P \leq$ $0.05)$. With the exception of cultivar by year, none of the interactions was statistically significant, indicating a single model would be meaningful for both cultivars. The quantitative relationship between percent maximum yield and disease was explained by the regression model $y=96.9-0.45 x$, in which the independent variable was BYD incidence assessed at growth stage 10.5. Disease incidence ranged from 0 to $95 \%$ in Noble and from 0 to $30 \%$ in Ogle (Table 1; Fig. 1). The overall $F$ value for this model was $79.1(P \leq 0.01)$. The coefficient of determination $\left(r^{2}\right)$ was 0.53 , and the regression coefficient $(-0.45)$ was significantly different from $0(t=-7.3 ; P \leq 0.01)$. The PRESS statistic was 131.09. This value is a reasonable estimate of the mean square error of 131.32. Residual plots of either $x$ or $\hat{Y}$ indicated no departures from normal. The model predicts that for each percent increase in BYD disease incidence, there will be a $0.45 \%$ decrease in yield for the two cultivars.

Compensation test. Three weeks after inoculation, distinct foci of disease could be seen in plots inoculated with the mechanical infestation device. Diseased plants were interspersed with healthy plants in plots that received the random inoculation treatment. Focus or random patterns of disease development were easily identified in plots of both trials throughout the tests. Visual assessments of disease at growth stage 10.5 are reported here, although disease assessments at stage 10.0 produced a similar result (data not shown).

As in the yield loss experiments, disease incidence was greater in 1992 than in 1991 (Table 2). Disease incidence ranged from 5 to $30 \%$ in 1991 and from 8 to $70 \%$ in 1992. Mean disease incidence was not affected by inoculation technique in either experiment. Yields ranged from 2,562 to $4,052 \mathrm{~kg} / \mathrm{ha}$ in 1991 and from 1,472 to $3,781 \mathrm{~kg} / \mathrm{ha}$ in 1992.

Plots inoculated with foci averaged 3,344 and 2,651 kg/ha in 1991 and 1992, respectively. Plots inoculated with the random inoculation technique averaged 3,299 and 2,231 kg/ha in 1991 and 1992, respectively.

In both trials, inoculation technique by disease incidence interactions were not statistically significant (Table 3). Therefore, yield loss attributable to BYD disease was not affected by inoculation method.

Both trials produced similar models, although the model produced from the DeKalb

Table 2. Mean and range of barley yellow dwarf (BYD) disease incidence and yield of oat cv. Noble in two experiments to compare the effect of random and focus inoculations with barley yellow dwarf virus

\begin{tabular}{|c|c|c|c|c|c|c|}
\hline \multirow[b]{2}{*}{ Experiment } & \multicolumn{3}{|c|}{ Disease incidence $(\%)^{\mathrm{a}}$} & \multicolumn{3}{|c|}{ Yield (kg/ha) } \\
\hline & $\begin{array}{l}\text { Focus } \\
\text { mean }^{b}\end{array}$ & $\begin{array}{c}\text { Random } \\
\text { mean }^{c}\end{array}$ & Range & $\begin{array}{l}\text { Focus } \\
\text { mean }\end{array}$ & $\begin{array}{c}\text { Random } \\
\text { mean }\end{array}$ & Range \\
\hline Urbana, IL, 1991 & 15 & 16 & $5-30$ & 3,344 & 3,299 & $2,562-4,052$ \\
\hline DeKalb, IL, 1992 & 28 & 36 & $8-70$ & 2,651 & 2,231 & $1,472-3,781$ \\
\hline
\end{tabular}

${ }^{a}$ Disease incidence was determined by visually estimating the percentage of plants in each plot with BYD symptoms at Feekes growth stage 10.5 .

${ }^{\mathrm{b}}$ Mean disease of plots inoculated by placing viruliferous aphids on two to three adjacent plants, creating disease foci.

${ }^{c}$ Mean disease of plots inoculated by shaking viruliferous aphids over the plot, creating a random pattern of disease.

Table 3. Analysis of covariance of two experiments to compare the effects of random and focus inoculations with barley yellow dwarf disease (BYD) on percent yield in oat cv. Noble

\begin{tabular}{lcc}
\hline Source of variation & \multicolumn{1}{c}{ Urbana, IL, 1991 } & DeKalb, IL, 1992 \\
\hline Block & $* * \mathrm{~d}$ & $\mathrm{NS}$ \\
Inoculation technique $^{\mathrm{b}}$ & $\mathrm{NS}$ & $\mathrm{NS}$ \\
Disease incidence $^{\mathrm{e}}$ & $* *$ & $* *$ \\
$\begin{array}{l}\text { Inoculation technique by disease } \\
\text { incidence interaction }\end{array}$ & $\mathrm{NS}$ & $\mathrm{NS}$ \\
Model produced & $y=100.0-0.61 x$ & $y=100.0-0.65 x$ \\
Student's $t$ value for slope estimate & -1.94 & $-6.38^{* *}$ \\
$r^{2}$ & 0.12 & 0.59 \\
\hline a Block and inoculation technique are class variables, and disease incidence is the covariate. \\
b Plots were inoculated by placing viruliferous aphids on two to three plants, creating foci of disease, \\
and by shaking viruliferous aphids over the plot, creating a random pattern of disease. \\
${ }^{c}$ Disease incidence was determined by visually estimating the percentage of plants in each plot with \\
BYD symptoms at Feekes growth stage 10.5. \\
d $*$ and $* *=$ significantly different from 0 at $P \leq 0.05$ and $P \leq 0.01$, respectively. \\
e Not significant $(P>0.05)$.
\end{tabular}


trial had a higher $r^{2}$ and a significant $t$ value for the test of the slope (Table 3). The slope in the Urbana trial was $-0.61(P \leq$ $0.10)$, and the slope in the DeKalb trial was $-0.65(P \leq 0.01)$. Both models estimated the intercept at $100 \%$.

\section{DISCUSSION}

The quantitative relationship between BYD disease incidence and oat yield was best explained by regression models in which the independent variable was BYD incidence assessed at growth stage 10.5. The best estimate of yield reduction due to BYD was approximately $4.5 \%$ for each $10 \%$ increase in BYD incidence.

The average yield of the cv. Ogle was higher in $1992(6,299 \mathrm{~kg} / \mathrm{ha})$ than in 1991 $(3,703 \mathrm{~kg} / \mathrm{ha})$, whereas the average yield of Noble was comparatively similar for both years $(3,724$ and $3,179 \mathrm{~kg} / \mathrm{ha}$, respectively) (Table 1). In 1991, there was no precipitation during the month of June, the critical time period for grain fill. This adversely affected the yield of both cultivars. In 1992, spring and summer precipitation was normal, and Ogle responded with high yields. BYD incidence on Noble averaged $15.0 \%$ in 1991 and $69.8 \%$ in 1992, whereas BYD incidence on Ogle averaged only 9.7 and $12.9 \%$ for the same time periods. The comparatively high level of disease incidence on Noble in 1992 would account for its low yield that year.

Nine plots of Noble in 1992 had BYD incidence greater than $60 \%$ (Fig. 1). Although these plots had high levels of disease incidence, they did not greatly affect the fit of the model. The PRESS statistic was particularly useful in determining whether the deletion of a few critical observations, such as those from plots with exceptionally high disease, impacted the model. The PRESS statistic was 131.09, and the mean square error was 131.32. These two values are almost identical, as would be expected if the nine plots had little effect on model estimates.

Once Ogle shows BYD symptoms, it does not tolerate BYD disease any better than Noble and sustains similar yield losses. Covariance analysis indicated that response to disease did not vary between the two cultivars, even though Noble sustained higher BYD incidence than Ogle throughout the study. It is interesting that slope estimates from the compensation tests with Noble alone are slightly lower $(-0.61$ and -0.65$)$ than those produced by the model with both cultivars (-0.45) (Fig. 1; Table 3). Because these slopes were generated in separate experiments, it is not possible to compare them directly. Examination of the standard errors of these slopes suggests they may be estimates of the same value.

Because the objective was to create a model that could be applied to a grower's field, no attempt was made to control natural aphid populations. Although insecticides were applied to control aphid populations used to initiate epidemics, additional infections by naturally occurring viruliferous aphids almost certainly occurred. Plants with characteristic symptoms could have been infected at different times as indigenous aphid populations moved and reproduced throughout the plots. This permitted the modeling of a dynamic epidemic. The age of a plant at the time of inoculation affects oat yield $(6,11)$. Generally, early infection results in greater yield loss and symptom expression than late infection. This may account for the variability in yield response to BYD incidence and the low $r^{2}$ associated with the yield loss model presented here.

No measurable effects were detected in tests to determine the impact of compensation on yield loss of Noble oats. There is no indication in these studies that inoculation methods and subsequent effects on the degree of compensation affected yield loss. Both methods of inoculation (focus and random) can be used in future yield loss studies.

\section{LITERATURE CITED}

1. Allen, D. M. 1971. The prediction sum of squares as a criterion for selecting predictor variables. Tech. Rep. 23. Department of Statistics, University of Kentucky, Lexington.

2. Bissonnette, S. M., D'Arcy, C. J., and Pedersen, W. L. 1994. Yield loss in two spring oat cultivars due to Puccinia coronata f. sp. avenae in the presence or absence of barley yellow dwarf virus. Phytopathology 84:363-371.

3. Conti, M., Jedlinski, H., and Burnett, P. A. 1990. The "yellow plague" of cereals, barley yellow dwarf virus. Pages 1-6 in: World Perspectives on Barley Yellow Dwarf. P. A. Burnett, ed. CIMMYT, D.F., Mexico.

4. D'Arcy, C. J., Hewings, A. D., and Eastman, C. E. 1992. Reliable detection of barley yellow dwarf viruses in field samples by mono- clonal antibodies. Plant Dis. 76:273-276.

5. D'Arcy, C. J., Torrance, L., and Martin, R. R. 1989. Discrimination among luteoviruses and their strains by monoclonal antibodies and identification of common epitopes. Phytopathology 79:869-873.

6. Doodson, J. K., and Saunders, P. J. 1970. Some effects of barley yellow dwarf virus on spring and winter cereals in field trials. Ann. Appl. Biol. 66:361-374.

7. Draper, N., and Smith, H. 1981. Applied Regression Analysis. 2nd ed. John Wiley \& Sons, New York.

8. Gildow, F. E., and Frank, J. A. 1988. Barley yellow dwarf virus in Pennsylvania: Effect of the PAV isolate on yield components of Noble spring oats. Plant Dis. 72:254-256.

9. Gill, C. C. 1967. Transmission of barley yellow dwarf virus isolates from Manitoba by five species of aphids. Phytopathology 57: 713-718.

10. Gill, C. C. 1980. Assessment of losses on spring wheat naturally infected with barley yellow dwarf virus. Plant Dis. 64:197-203.

11. Goulart, L. R., Ohm, H. W., and Foster, J. E. 1989. Barley yellow dwarf symptom severity in oat affected by plant growth stage at infection and plot type. Crop Sci. 29:14121416.

12. Hewings, A. D., Kolb, F. L., Gregerson, G. R., and Bauske, E. M. 1992. Field research and germplasm evaluation methodology for barley yellow dwarf virus in cereals. A. R. Barr, A. R. Barr, R. J. McLean, J. D. Oates, G. Roberts, J. Rose, K. Saint, and S. Tasker, eds. Proc. 4th Int. Oat Conf. 3:44-46.

13. James, W. C. 1974. Assessment of plant diseases and losses. Annu. Rev. Phytopathol. 12 27-48.

14. Large, E. C. 1954. Growth stages in cereals. Plant Pathol. 3:128-129.

15. Littell, R. C., Freund, R. J., and Spector, P. C. 1991. SAS System for Linear Models. 3rd ed. SAS Institute, Cary, NC.

16. Plumb, R. T. 1983. Barley yellow dwarf virusA global problem. Pages 185-188 in: Plant Virus Epidemiology. R. T. Plumb and J. M. Thresh, eds. Blackwell Scientific Publication, London.

17. Reestman, A. J. 1970. Importance of the degree of virus infection for the production of ware potatoes. Potato Res. 13:248-268.

18. Rochow, W. F. 1969. Biological properties of four isolates of barley yellow dwarf virus. Phytopathology 59:1580-1589.

19. Smith, P. R., and Sward, R. J. 1982. Crop loss assessment studies on the effects of barley yellow dwarf virus in wheat in Victoria. Aust. J. Agric. Res. 33:179-185.

20. University of Illinois. 1992. Illinois Agronomy Handbook. Coll. Agric. Coop. Ext. Ser. Circ. 1311.

21. Wiseman B. R., Davis, F. M., and Campbell, J. E. 1980. Mechanical infestation device used in fall armyworm plant resistance programs. Fla. Entomol. 63:425-431. 